\title{
The Health Status of 50-70 Years Old Women in Some Areas - China, 2018
}

\author{
Bo Songl ${ }^{1,8}$; Gengli Zhao ${ }^{2,8 \times}$; Jiangli $\mathrm{Di}^{1, *,}$; Yu Ma ${ }^{1}$; Linhong Wang ${ }^{3, *}$
}

\section{Summary \\ What is already known about this topic?}

After menopause, women are prone to chronic diseases such as cardiovascular disease, diabetes and osteoporosis and gynecological diseases such as pelvic floor dysfunction and reproductive tract infection.

What is added by this report?

The prevalence of hypertension, diabetes, cardiovascular disease and osteoporosis increased with age, while the prevalence of vaginitis and cervicitis decreased with the increase of age. The proportions of women aged 50-70 years old suffering from chronic and gynecological diseases in western and rural areas were significantly higher than those in eastern, central and urban areas.

What are the implications for public health practice?

It is necessary to take targeted intervention measures in the western region and rural areas, in order to narrow the gap in health of menopausal and older women between different areas of China.

The number of women over the age of 50 in China is estimated to increase to more than 280 million by 2030 (1). With an increase in age, the mortality rate of people aged 50 and above in China is rising rapidly, and the prevalence of chronic diseases in middle-aged and older people (45 years old and above) is much higher than that in other age groups (2). With the aging of the population, the health of menopausal and older women has become prioritized. The purpose of this study is to investigate the health status of menopausal and older women in different areas of China through a survey of women aged $50-70$ years to provide a scientific basis for promoting the health of women. A cross-sectional survey involving 5,049 women aged 50-70 years was conducted across eastern, central, and western China. The main result of this study showed that the health problems of menopausal and older women in the western region and rural areas were more prominent. Taking effective measures is necessary to narrow the gap in health between different age groups of women and different regions.

This study was a survey of women aged $50-70$ years in the National Survey of Women's Health conducted in 2018. The national survey was conducted to represent the three socioeconomic regions of China: eastern (Jiangsu and Shandong provinces), central (Hunan and Anhui provinces), and Western (Shaanxi and Sichuan provinces). In each province, one urban and one rural area were selected as investigation sites. A total of 5,049 women aged 50-70 years were selected by multistage stratified random cluster sampling and completed face-to-face interview questionnaires in the national survey. This study analyzed the relevant survey results of women aged 50-70 years. The reported chronic diseases and gynecological diseases in this study were required to be diagnosed by secondary hospitals or above.

The average age of the respondents was $58.94 \pm 6.195$ years. Among the respondents, 1,703 (33.7\%) came from the eastern region, $1,667(33.0 \%)$ from the central region, and 1,679 (33.3\%) from the western region. Most respondents (85.5\%) were married, and $11.7 \%$ were widowed. The main occupation of the cohort was farming, accounting for $55.2 \%$, followed by retirees, accounting for $17.1 \%$. Most of the participants $(55.0 \%)$ had only a primary school education or were illiterate, followed by those who had junior and senior high school educations, accounting for $42.0 \%$. Only $3.0 \%$ had junior college degrees or higher. The majority (89.9\%) of the women were postmenopausal.

Among the 5,049 women, $55.1 \% \quad(95 \%$ CI: $53.7 \%-56.5 \%)$ reported that they had chronic diseases diagnosed by the hospital. The proportions of women suffering from chronic diseases in the western region (58.5\%, 95\% CI: $56.2 \%-60.9 \%)$ and rural areas (57.8\%, 95\% CI: $55.9 \%-59.7 \%)$ were higher than that in the eastern $(51.8 \%, 95 \%$ CI: $49.4 \%-54.2 \%)$, central $(55.0 \%, 95 \%$ CI: $52.6 \%-57.4 \%)$, and urban areas $(52.5 \%, 95 \%$ CI: $50.5 \%-54.4 \%)$. There were differences between different regions and areas 
$(p<0.001)$. The proportion of self-reported overweight and obesity was the highest $(46.5 \%, 95 \% \mathrm{CI}$ : $45.0 \%-47.9 \%)$. The prevalence of overweight and obesity among women in the eastern region $(55.8 \%$, 95\% CI: $53.3 \%-58.2 \%$ ) was significantly higher than the western $(45.4 \%, 95 \%$ CI: $42.7 \%-48.0 \%)$ and central regions $(37.8 \%$, 95\% CI: $35.4 \%-40.3 \%)$ $(p<0.001)$. The prevalence of cardiovascular disease $(11.9 \%, \quad 95 \%$ CI: $10.4 \%-13.5 \%)$, osteoporosis $(10.9 \%, 95 \%$ CI: $9.4 \%-12.4 \%)$, and kidney disease $(2.6 \%, 95 \%$ CI: $1.9 \%-3.4 \%)$ among women in the western region were significantly higher than those in the eastern and central regions $(p<0.005)$. The prevalence of osteoarthrosis $(15.0 \%, 95 \% \mathrm{CI}$ : $13.6 \%-16.4 \%)$ in rural women was higher than that of urban women $(11.4 \%$, 95\% CI: $10.1 \%-12.6 \%)$. However, the prevalence of osteoporosis $(9.8 \%, 95 \%$ CI: $8.6 \%-11.0 \%)$, oral disease $(2.6 \%, 95 \%$ CI: $2.0 \%-3.2 \%)$, and thyroid disease $(1.6 \%, 95 \% \mathrm{CI}$ : $1.1 \%-2.1 \%$ ) in urban women were higher than those in rural women $(5.7 \%, 95 \%$ CI: $4.8 \%-6.6 \%, 1.3 \%$, $95 \%$ CI: $0.9 \%-1.8 \%$, and $0.8 \%, 95 \%$ CI: $0.4 \%-1.1 \%$, respectively) $(p<0.01)$ (Table 1 ).

Of the participating women, $39.6 \%(95 \% \mathrm{CI}$ : $38.3 \%-40.9 \%)$ reported that they had gynecological diseases diagnosed by the hospital. The proportion of women suffering from gynecological diseases in the central $(42.6 \%, 95 \%$ CI: $40.2 \%-45.0 \%)$, western $(42.7 \%$, 95\% CI: $40.3 \%-45.1 \%)$, and rural areas (42.2\%, 95\% CI: $40.3 \%-44.1 \%)$ was higher than that in the eastern $(33.6 \%, 95 \%$ CI: $38.3 \%-40.9 \%)$ and urban areas $(37 \%, 95 \%$ CI: $35.1 \%-38.9 \%)$ $(p<0.001)$ ( Table 2).

Among the self-reported gynecological diseases, the prevalence of vaginitis was the highest $(24.4 \%, 95 \%$ CI: $23.2 \%-25.6 \%)$. The prevalence of vaginitis (29.4\%, 95\% CI: $27.2 \%-31.5 \%)$ and pelvic inflammation $(9.2 \%, 95 \% \mathrm{CI}: 7.8 \%-10.6 \%)$ in the western region were significantly higher than those in the eastern region $(p<0.001)$. The prevalence of urinary incontinence in the central region $(14.6 \%, 95 \% \mathrm{CI}$ : $12.9 \%-16.3 \%)$ was significantly higher than that in the eastern region $(6.3 \%, 95 \%$ CI: $5.1 \%-7.4 \%)$ $(p<0.001)$. The prevalence of benign tumors in the eastern region $(12.0 \%, 95 \% \mathrm{CI}: 10.5 \%-13.6 \%)$ was significantly higher than that in the western region $(8.9 \%, \quad 95 \%$ CI: $7.6 \%-10.3 \%) \quad(p<0.001)$. The prevalence of vaginitis $(29.0 \%, 95 \% \mathrm{CI}$ : $27.2 \%-30.7 \%)$ and cervicitis $(13.7 \%, 95 \%$ CI: $12.3 \%-15.0 \%)$ in rural women were significantly higher than those in urban women $(19.8 \%, 95 \% \mathrm{CI}$ :
$18.3 \%-21.4 \%$ and $10.2 \%, 95 \%$ CI: $9.0 \%-11.4 \%$, respectively) $(p<0.001)$ (Table 2$)$.

The prevalence of hypertension, diabetes, cardiovascular disease, osteoporosis, and urinary incontinence increased with age, and there were significant differences among different age groups $(p<0.001)$. The prevalence of vaginitis, cervicitis and gynecological benign tumor was the highest in the age group of 50-54 years old. The prevalence of vaginitis and cervicitis decreased with increasing age, and there were significant differences among different age groups $(p<0.001)$ (Table 3).

\section{DISCUSSION}

Chronic non-communicable diseases in menopausal and older women seriously affected their health. The literature showed that the prevalences of hypertension, diabetes, hyperlipidemia, and cardiovascular disease in women over 50 years old were significantly higher than those below 50 years old, and the risk of cardiovascular and cerebrovascular diseases was close to or even higher than that in men in a short period of time (3-4). In this study, the proportion of women aged $50-70$ years old with chronic diseases was $55.1 \%$. Overweight and obesity (46.5\%), hypertension (28.5\%), osteoarthrosis $(13.2 \%)$, diabetes $(10.2 \%)$, cardiovascular disease (9.8\%), and osteoporosis (7.8\%) were major health problems for menopausal and older women. Moreover, the prevalence of chronic diseases increased gradually with age. The prevalence of chronic diseases in the age group of 50-54 years old was $46.3 \%$, and it had increased to $68.2 \%$ in $65-70$ age group. However, the prevalence was lower than the results of China's Fifth National Health Service Survey in 2013 (the prevalence of chronic disease in women aged 55-64 years old was $57.0 \%$ and that in women aged 65 or above was $82.9 \%$ ) (5). The reason may be related to the strengthening of health management of the elderly in recent years, increased availability of free physical examinations, and the awareness of the healthy lifestyle of older women. Therefore, in order to improve the health of the elderly, the health management of the elderly should be further strengthened and their health awareness should be improved.

The prevalence of gynecological diseases in menopausal women was higher. This study found that $39.6 \%$ of the women were diagnosed with gynecological diseases, and the proportion of gynecological diseases in the 50-54 age group was the highest $(47.9 \%)$. Most of them were reproductive tract 

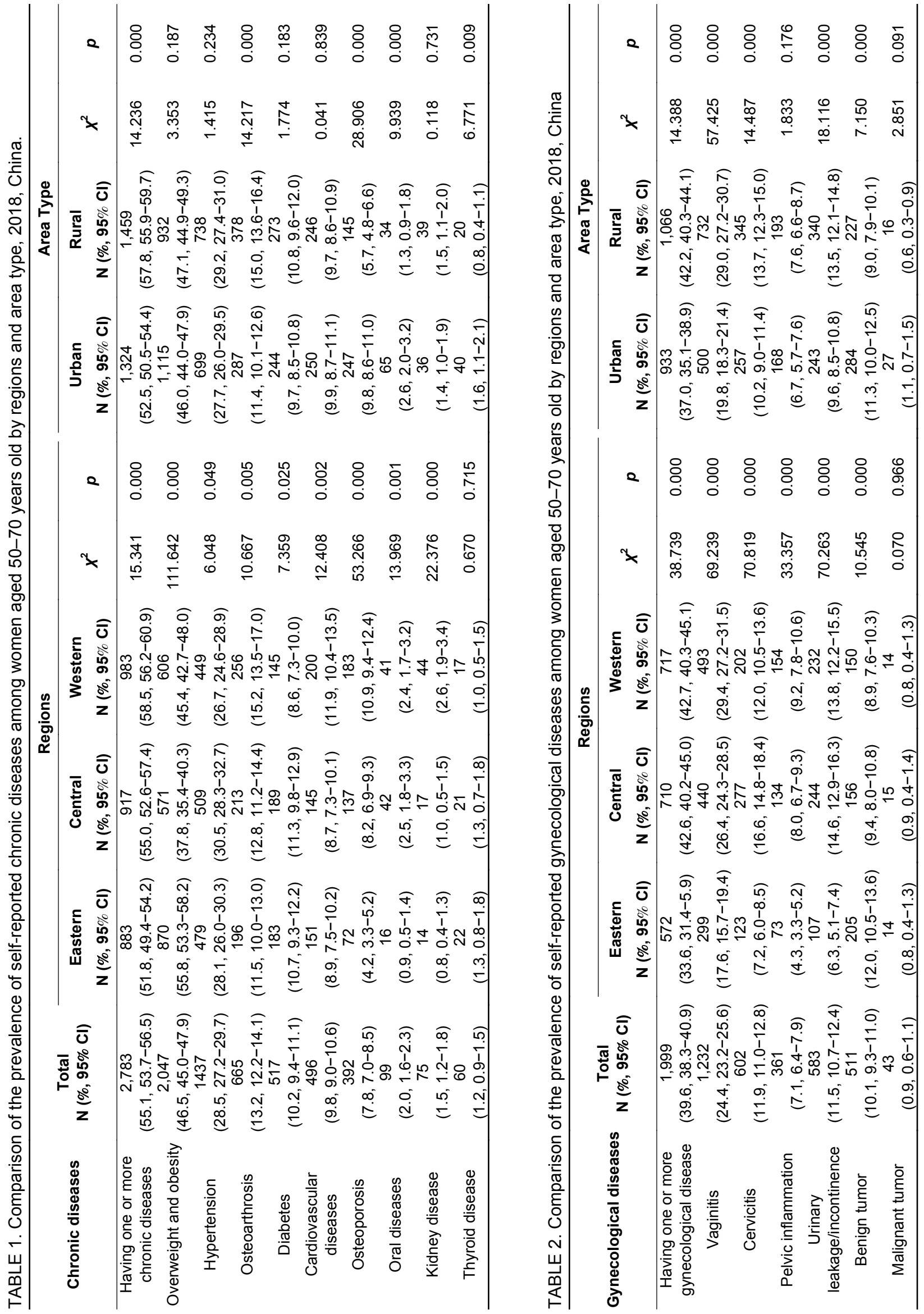


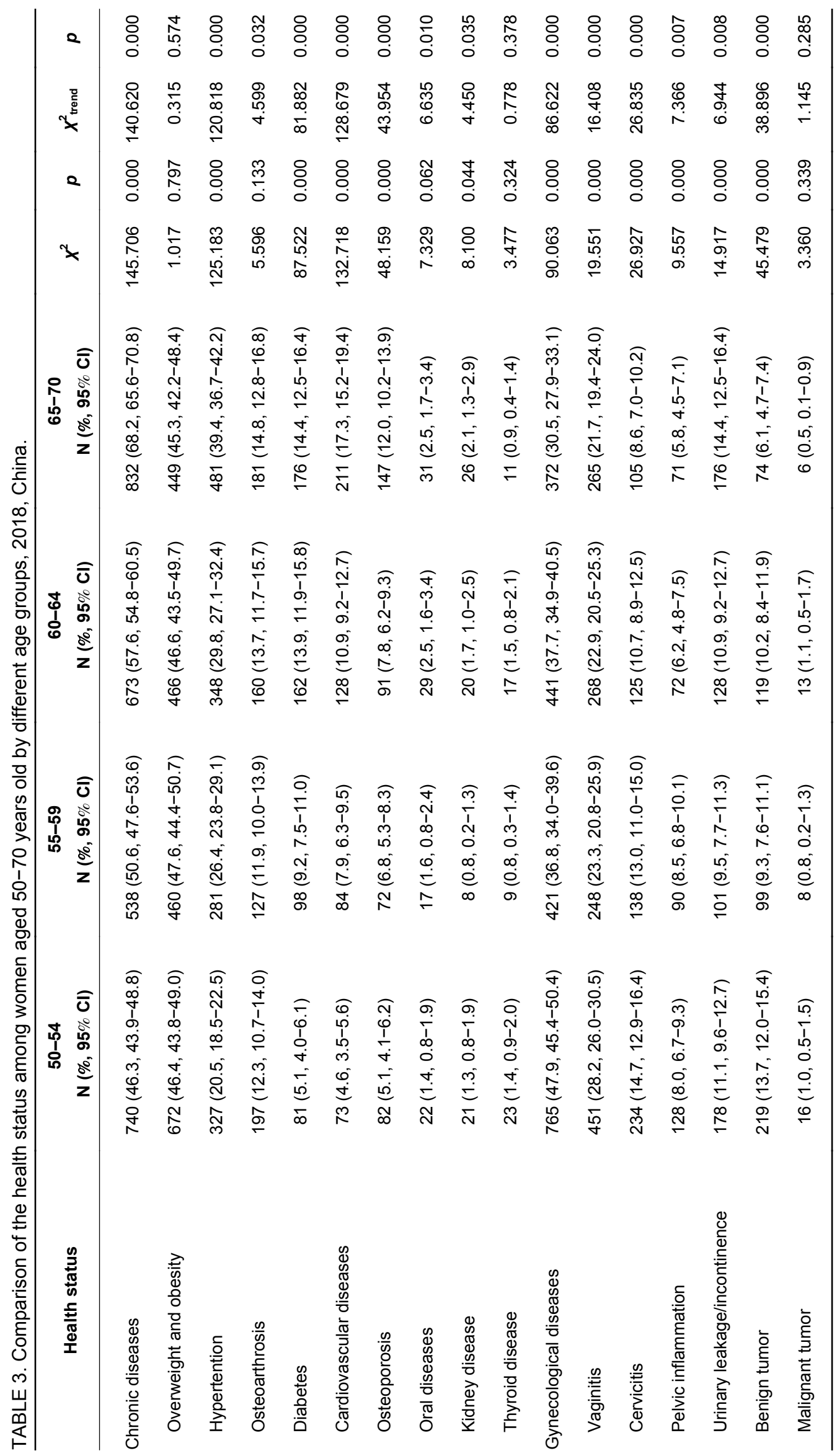


infections (such as vaginitis, cervicitis, and pelvic inflammation). This result is further verified because the decline of ovarian function in menopausal women leads to genitourinary tract atrophy, and they are more prone to reproductive tract infection (G).

The health problems of menopausal and older women in the western region and rural areas were more prominent. Another important result of this study was that an imbalance of regional development was an important factor restricting the health of menopausal and older women. Compared with the eastern and urban areas, the proportions of women suffering from chronic and gynecological diseases in the western and rural areas were significantly higher. However, China's Fifth National Health Service Survey in 2013 showed that in recent years, the prevalence of chronic diseases among urban and rural residents has increased rapidly, and the growth rate in rural areas was higher than that in urban areas (5). The reason may be due to the gradual economic development and the improvement of living standards in rural and western areas, but the population lacks the awareness of active health and the ability of self-health management and the health care service and infrastructure are relatively insufficient. Therefore, the health problems of menopausal and older women in the western region and rural areas are more prominent and need to more attention. The construction of infrastructure, especially medical and health-related facilities, should be improved and the conditions of health care services should be strengthened in the western region and rural areas. In order to achieve the goal of "Joint construction and sharing, health for all" in the outline of the Plan of Healthy China 2030, the imbalance in regional development should be mitigated and eliminated as soon as possible.

This study was subject to at least some limitations. First, the clinical diagnosis was self-reported and might be subjected to biases. Second, although the age composition of the participants in this study was similar to that of women in the 2018 national population sampling survey (the difference of age composition ratio ranged from $0.36 \%$ to $4.88 \%$ ) (7), the study used convenience sampling, and data were collected in 12 counties/districts in 6 provinces, so the results might not fully be representative of the regional and national levels.

doi: $10.46234 / \mathrm{ccdcw} 2020.142$

\# Corresponding authors: Jiangli Di, dijiangli@chinawch.org.cn; Linhong Wang, linhong@chinawch.org.cn.

\footnotetext{
'National Center for Women and Children's Health, Chinese Center for Disease Control and Prevention, Beijing, China; ${ }^{2}$ Women and Children's Health Center of the First Hospital of Peking University, Beijing, China; ${ }^{3}$ National Center for Chronic and Non-communicable Disease Control and Prevention, Chinese Center for Disease Control and Prevention, Beijing, China.

\& Joint first authors.
}

Submitted: June 20, 2020; Accepted: July 03, 2020

\section{REFERENCES}

1. Women's Health Branch of Chinese Medical Association, Climacteric Health Care Group. Menopausal women's health care guide (2015). J Pract Gynecol Endocrinol, 2016;3(2):21 - 32. doi: 10.16484/j. cnki.issn2095-8803.2016.02.012. (In Chinese).

2. Li YN. Study on functional impairment, quality of life and its influencing factors in people aged 50 and over in China[dissertation]. Beijing: Chinese Center for Disease Control and Prevention; 2019. https://kns.cnki.net/KCMS/detail/detail.aspx?dbcode=CMFD\&dbname CMFD201902\&filenam=1019196033.nh\&vMTI4MjdmWU9adUZpR GdWN3ZQVkYyNkY3S3hHTkhQckpFYlBJUjhlWDFMdXhZUzdEa DFUM3FUcldNMUZyQ1VSN3E=. (In Chinese).

3. Disease Prevention and Control Bureau of the National Health and Family Planning Commission. Report on nutrition and chronic diseases of Chinese residents. Beijing: People's Medical Publishing House. 2015. (In Chinese).

4. Savonitto S, Colombo D, Franco N, Misuraca L, Lenatti L, Romano IJ, et al. Age at menopause and extent of coronary artery disease among postmenopausal women with acute coronary syndromes. Am J Med, 2016, 129(11):1205 - 12. https://www.sciencedirect.com/science/article/ abs/pii/S0002934316305642.

5. Statistical Information Center of the National Health and Family Planning Commission. Fifth national health service survey and analysis report. Beijing: China Union Medical University Press, 2013. http:// www.nhc.gov.cn/mohwsbwstjxxzx/s8211/201610/9f109ff40e9346fca76d d82cecf419ce.shtml. (In Chinese).

6. Zhu ZY, Li CB, Tao MF. Characteristics of menopausal symptoms in menopausal women and its influencing factors. J Endocr Surg 2016;10(4):320 - 4, 39. https://kns.cnki.net/KCMS/detail/detail.aspx? dbcode $=$ CJFD \&dbname $=$ CJFDZHYX\&filename $=$ NFMW201604016\& v=Mjg1Nzk4ZVgxTHV4WVM3RGgxVDNxVHJXTTFGckNVUjdxZl 1PWnVGaURrV3I3Tkt5dkdlYkc0SDlmTXE0OUVZb1I=. (In Chinese)

7. National Bureau of Statistics. China statistical yearbook (2019). Beijing. China Statistics Publishing House, 2019. (In Chinese). 